\title{
Simulação médica no período da pandemia da COVID-19: um relato de experiência
}

\author{
Medical simulation in the COVID-19 pandemic period: an experience report
}

\author{
Medical simulación medica en el periodo de la pandemia del COVID-19: un informe de \\ experiencia
}

Gustavo Garcia Pacheco1*, Mariana Scapellato Gontijo1, Letícia Donaire Fabro1, Maria Laura Verissimo Teixeira$^{1}$, Carolina Marques Ferreira ${ }^{1}$, Jhenifer Rodrigues Messias ${ }^{1}$, Maíra Ferro de Sousa Touso ${ }^{1}$.

\section{RESUMO}

Objetivo: Apresentar e refletir o emprego remoto da metodologia "Debriefing" utilizada na simulação em prática médica, nas aulas remotas durante a pandemia do novo coronavírus. Relato de experiência: Trata-se de um relato de experiência conduzido por seis acadêmicos de medicina do sexto período na disciplina de Programa de Integração na Estratégia Saúde da Família no estágio de saúde mental. O estágio foi vivenciado por seis alunos e devido às restrições impostas pela pandemia do novo coronavírus todas as aulas foram realizadas de maneira remota. O programa da disciplina foi composto de quatro encontros para discussão dos principais temas da Psiquiatria seguido de três encontros no qual a metodologia Debriefing foi aplicada. A cada encontro um caso clínico era trazido pelo professor que além de conter informações da anamnese e do exame físico, possuía pequenos vídeos com simulações realísticas do caso apresentado. Após a apresentação do quadro do paciente fictício o professor orientava o processo de construção de aprendizagem por meio de perguntas nas quais os alunos teriam que utilizar de conhecimentos teóricos para respondê-las. Considerações finais: Essa ferramenta se mostrou eficaz no contexto de aulas síncronas durante a pandemia da COVID-19.

Palavras-chaves: Debriefing, Covid-19, Educação médica.

\section{ABSTRACT}

Objective: To present and reflect the remote use of the "Debriefing" methodology used in simulation in medical practice, in remote classes during the pandemic of the new coronavirus. Experience report: This is an experience report conducted by six medical students from the sixth period in the Integration Program discipline at Family Health Strategy at the mental health stage. The internship was experienced by six students and due to the restrictions imposed by the pandemic of the new coronavirus, all classes were held remotely. The discipline program consisted of four meetings to discuss the main themes of Psychiatry followed by three meetings in which the Debriefing methodology was applied. At each meeting, a clinical case was brought by the teacher who, in addition to containing information from the anamnesis and physical examination, had short videos with realistic simulations of the case presented. After presenting the picture of the fictitious patient, the teacher guided the process of building learning through questions in which students would have to use theoretical knowledge to answer them. Final considerations: This tool proved to be effective in the context of synchronous classes during the COVID-19 pandemic.

Keywords: Debriefing, Covid-19, Medical education.

\section{RESUMEN}

Objetivo: Presentar y reflejar el uso a distancia de la metodología "Debriefing" utilizada en simulación en la práctica médica, en clases remotas durante la pandemia del nuevo coronavirus. Informe de experiencia: Se trata de un informe de experiencia realizado por seis estudiantes de medicina del sexto período en la disciplina del Programa de Integración en la Estrategia de Salud de la Familia en el área de salud mental. etapa. La pasantía la vivieron seis estudiantes y debido a las restricciones impuestas por la pandemia del nuevo coronavirus, todas las clases se realizaron de forma remota. El programa de disciplina consistió en cuatro reuniones para discutir los principales temas de Psiquiatría seguidas de tres reuniones en las que se aplicó la metodología Debriefing. En cada encuentro, el docente trajo un caso clínico quien, además de contener información de la anamnesis y el examen físico, contó con videos cortos con simulaciones realistas del caso presentado. Luego de presentar la imagen del paciente ficticio, el docente guió el proceso de construcción del aprendizaje a través de preguntas en las que los estudiantes tendrían que usar conocimientos teóricos para responderlas. Consideraciones finales: Esta herramienta demostró ser efectiva en el contexto de clases sincrónicas durante la pandemia de COVID-19.

Palabras clave: Debriefing, Covid-19, Educación médica.

\footnotetext{
${ }^{1}$ Universidade de Franca (UNIFRAN), Franca - SP. *E-mail: garciagustavopacheco@gmail.com
} 


\section{INTRODUÇÃO}

No final da década de 1980 a literatura anestésica foi a primeira a descrever a simulação médica como ferramenta para o ensino (PATTI L e SCHERTZER K, 2020). Atualmente essa técnica é um elemento importante para o processo de aprendizagem e é incorporada como estratégia de ensino pelas instituições de graduação e pós-graduação (GRANT VJ, et al., 2018). Vale ressaltar que a experiência é apenas o ponto inicial, sendo necessário uma reflexão sobre a vivência para de fato concretizar o estudo (BOWE SN, et al., 2017). O debriefing é o momento dos participantes refletirem sobre os acontecimentos da sessão e como resultado, alterar sua conduta nas sessões subsequentes (JOHNSTON S, et al., 2018).

Existem vários tipos de simulação: simulação tradicional: é aquela realizada em laboratórios de simulação realística; simulação in situ: é aquela que acontece no ambiente clínico real. Esse tipo de simulação oferece como diferencial o ensino a nível organizacional; simulação remota: é aquela que ocorre em ambiente virtual, por meio de vídeos (PATTI L e SCHERTZER K, 2020).

O debriefing pode ocorrer no final da simulação ou em pontos de parada frequentes durante uma simulação. $O$ facilitador instrui os participantes durante o processo reflexivo e procuram constatar e superar as lacunas de conhecimento por meio da discussão (ALI AA e MILLER ET, 2018). A discussão dirigida possui vantagens sobre a reflexão autodirigida. No sentido amplo, aprendizagem autodirigida descreve o processo no qual os indivíduos tomam a iniciativa sem a ajuda de outros para diagnosticar as suas necessidades de aprendizagem. Neste processo as conclusões podem ser assistemáticas e até mesmo ausentes. Por outro lado, quando um problema é apresentado pelo professor e todos os alunos o discutem sob sua orientação todos os alunos são submetidos aos mesmos pontos de reflexão de modo sistemático, permitindo com que os objetivos de aprendizado sejam cumpridos (PATTI L e SCHERTZER K, 2020).

O debriefing pode ser executado de modo oral, escrito ou por vídeo. O debriefing oral é baseado em perguntas do instrutor aos participantes, sendo necessário apenas que o aluno responda verbalmente as indagações (ALI AA e MILLER ET, 2018). Já o debriefing escrito é baseado na entrega de um questionário para cada aluno ao final da simulação, na qual deve respondido por escrito todas as perguntas. Por fim, 0 debriefing por vídeo parte do pressuposto de gravar o momento da simulação e posteriormente assistir essas filmagens discutindo os pontos pertinentes (FELIX HM, et al., 2020).

Um dos benefícios da utilização do vídeo assistido é a obtenção de um registro fidedigno aos acontecimentos reais. Um debriefing limitado ao viés da memória e sem o registro concreto pode ser prejudicado ao ser discutido apenas o que foi recordado pelos participantes. Além disso, o acesso as simulações permitem uma reflexão por parte do participante sobre o seu desempenho durantes os eventos, trazendo confiança nas suas performances de sucesso e projeção para mudanças na identificação das deficiências (ALI AA e MILLER ET, 2018).

O debriefing assistindo por vídeo tem maior qualidade no que tange a preencher as lacunas de aprenzidado que o debriefing apenas verbal. Entretanto, possui algumas desvantagens: a necessidade de um suporte técnico para evitar problemas que prejudiquem as gravações e o receio por parte dos participantes que o material seja exibido fora da classe. Os resultados das comparações do debriefing por vídeo e debriefing verbal são mistos. Em um estudo com residentes de anestesiologia, nenhum grupo se mostrou superior ao outro. Mas ambos apresentaram melhores resultados que o grupo dos participantes que não tiveram 0 debriefing (ALI AA e MILLER ET, 2018).

Algumas situações podem comprometer a qualidade do debriefing: seja por fatores específicos relacionados ao aluno, seja por fatores relacionados à execução do debriefing (HARDER N e NUNES S, 2019). No primeiro caso, a ausência do domínio teórico, a timidez, o desinteresse, a aversão a críticas construtivas ou até mesmo alguma barreira linguística pode contribuir para um debriefing de má qualidade (OSTA AD, et al., 2019).

Já os fatores relacionados à execução incluem ausência de um suporte técnico durante as gravações e preparação inadequada do facilitador em relação a discussão e aos objetivos de aprendizagem (GRANT VJ, 
et al., 2018; TWIGG S, 2020). Um debriefing mal executado, que seja recebido pelo aluno como uma crítica não construtiva ao invés de construtiva pode criar rivalidades e resistência, consequentemente prejudicar 0 trabalho em equipe (ALI AA e MILLER ET, 2018). Sem o debriefing os erros cometidos durante a simulação costumam se perpetuar, o que consiste em um ponto negativo no processo de ensino (GURUAJA RP, et al., 2008).

O objetivo do presente trabalho é compreender o impacto do emprego de simulação médica remota com debriefing, nas atividades durante a pandemia da COVID-19.

\section{RELATO DE EXPERIÊNCIA}

O curso de Medicina da Universidade de Franca tem sua estrutura dividida em três fases. A primeira fase compreende o ciclo básico composto por quatro períodos, no qual é apresentado aos alunos a base do conhecimento médico. Seguidos por quatro períodos de ciclo clínico nos quais o foco é utilizar os conhecimentos básicos para aplicar no estudo das doenças. Por fim, os quatro últimos períodos são caracterizados pela imersão do aluno na realidade médica: o internato.

O Programa de Integração na Estratégia Saúde da Família (PIESF) é um módulo transversal presente do primeiro ao oitavo período. O módulo PIESF VI ocorreu durante o segundo semestre de 2020 e foi composto por dois eixos temáticos: saúde mental institucional e saúde mental clínica. Todas as aulas foram realizadas pela plataforma blackboard, devido ao contexto da pandemia, porém fora desse contexto as aulas seriam teóricas e práticas.

As aulas do PIESF VI em condições normais, ou seja, fora da circunstância de isolamento social imposto pela pandemia, ocorriam nas dependências do CAPs III da cidade de Franca, bem como no Hospital Psiquiátrico Allan Kardec, sob supervisão do médico psiquiatra preceptor responsável pela disciplina. Em média eram feitas quatro visitas nas quais o grupo de alunos eram divididos em duplas e tinham por objetivo realizar o atendimento dos pacientes. Ao final do atendimento os alunos precisavam apresentar ao preceptor a anamnese, a descrição do exame físico, uma hipótese diagnóstica e a conduta mais apropriada para o caso. As condutas eram discutidas amplamente com o preceptor e no final o mesmo explicava qual era a conduta mais adequada para seguir naquele caso. Por fim, então era realizada a confecção da receita pelos alunos e no final a mesma era revisada e assinada pelo professor.

A pesquisa foi realizada por seis acadêmicos de medicina, graduandos do sexto período, após analisarem as aulas realizadas de setembro a novembro. O presente trabalho é fruto da análise da metodologia utilizada nessas aulas de saúde mental clínica. Ao longo do semestre, foram realizados oito encontros, com a finalidade de se aprofundar nos seguintes tópicos: transtorno de humor, transtorno psicótico e transtorno de ansiedade. Em um primeiro momento os seis alunos foram divididos em duplas, totalizando três duplas. No primeiro encontro cada dupla ficou responsável por apresentar um tema relacionado a anamnese e exame psíquico. Do segundo encontro em diante cada dupla teria todo o período de uma aula para apresentar um seminário.

Os temas dessa apresentação foram: transtorno de humor, transtornos de ansiedade e esquizofrenia, que foram apresentados nessa ordem. Os alunos responsáveis pelo tema tinham como objetivo revisar a literatura sobre o tema e confeccionar uma aula para o grupo. Os descritores que foram avaliados nessa aula foram: os slides, o domínio do conteúdo e a capacidade de interação com os ouvintes. Para maior aproveitamento, todos os alunos dispuseram casos clínicos ao final de suas apresentações de modo a estimular o grupo a identificar os pontos apresentados na palestra em uma situação do dia-a-dia. Essa iniciativa dos alunos colaborou positivamente para o ótimo desempenho do grupo nas etapas subsequentes.

Posteriormente, foram realizados mais três encontros em que foram colocados em prática a metodologia de aprendizagem debriefing. Nessa nova conformação de aula, o professor trazia um caso clínico de um assunto que já havia sido discutido em encontros anteriores e o projetava em slides. Além dos slides, que continha a descrição da anamnese e do exame físico, eram reproduzidos pequenos vídeos que continham uma encenação de dois personagens em um consultório médico. Um dos personagens exercia o papel do médico e o outro encenava o paciente com sinais e sintomas característicos de um determinado distúrbio psiquiátrico. 
Após a apresentação do caso e conforme eram exibidos os vídeos, o professor designava um aluno para responder algumas questões presentes nos slides referentes a encenação, como a conduta do médico, sinais e sintomas do paciente, ambiente da consulta, aplicar exame psíquico no paciente, entre outros. Assim que o aluno respondia às indagações, o professor questionava ao grupo se mais alguém teria algo a acrescentar sobre aquele tópico. Por fim, o professor prontamente corrigia todas as perguntas feitas, e emitia um feedback imediato sobre as considerações dos alunos.

\section{DISCUSSÃO}

Os quatro primeiros encontros na qual foi estudado todo o conteúdo na forma de seminário foi determinante para o bom desenvolvimento das atividades posteriores de simulação e debriefing, uma vez que a prática simulada demanda um bom conhecimento prévio dos temas que serão abordados (BOWE SN, et al., 2017). Outro momento que contribuiu para a construção desse conhecimento prévio foram as sessões tutoriais sobre Saúde Mental durante o segundo semestre de 2020. Tal fato reafirma um dos pilares de um curso de metodologia ativa, como o PBL: a aquisição gradual do conhecimento por meio de disciplinas diferentes que abordam a mesma temática, proporciona ao aluno maior contato com o mesmo conteúdo, aumentando assim, maior possibilidade de compreensão e aprendizagem. Assim, as práticas simuladas desenvolvidas no PIESF ajudaram a concretizar conhecimentos já trabalhados pelos alunos em outras disciplinas.

Nos últimos três encontros, a simulação era feita baseada em vídeos que tinham em média um minuto de duração e várias características, tais como sinais e sintomas, características comportamentais e físicas do paciente, adequação da sala ao atendimento, e postura do médico, tiveram que ser extraídas, o que levou aos alunos a aprimorarem as habilidades de leitura verbal, não verbal e senso crítico em relação à atuação do médico ator. Além disso, sempre era solicitado que os alunos realizassem o exame psíquico, o que colaborou para que os alunos praticassem a aplicação dessa ferramenta fundamental nos atendimentos em psiquiatria. Tendo em vista a Taxonomia de Bloom, modelo educacional proposto para organizar de forma hierárquica as etapas do conhecimento por meio de níveis de complexidade e especificidade, os seis níveis do domínio cognitivo foram aplicados durante as aulas: conhecimento, compreensão, aplicação, análise, síntese e avaliação (BELHOT RV e FERRAZ APCM, 2010).

Outrossim, as discussões pós-simulação em grupo foi um fator contribuinte para o desenvolvimento das habilidades de comunicação, visto que o aluno tinha que exercitar sua síntese e o ordenamento lógico de ideias para construção de um bom raciocínio clínico. Para a construção do raciocínio clínico era utilizado o tripé diagnóstico baseado na anamnese, exame físico e exames complementares. Consequentemente ao desenvolvimento de competências de comunicação, o grupo se tornou mais coeso, fortalecendo o trabalho em grupo, tendo em vista que ao final todos tinham que expor sua opinião e entrar em um consenso sobre a melhor conduta para o caso (ALI AA e MILLER ET, 2018).

Além disso, a partir do conhecimento partilhado nas discussões o professor conseguia identificar falhas e promover o feedback instantâneo, o que ajudou os alunos a reconhecerem pontos que precisavam ser reforçados posteriormente (BUI AH, et al., 2021; CONOSCENTI E, et al., 2021). Tal fato contribuiu para o desenvolvimento da busca ativa do conhecimento, reforçando a autonomia do aluno no processo de aprendizagem, característica essencial a ser desenvolvida para a formação profissional. Essas lacunas de conhecimento deveriam ser revistas pelo aluno para a próxima aula e antes de dar início a um novo caso todas as dúvidas eram esclarecidas (BOWE SN, et al., 2017).

Ademais, ao final da simulação o grupo de alunos tinha que decidir por qual conduta tomar com o paciente, seja optar por continuar com consultas ambulatoriais ou promover a internação nos casos de urgência e de emergência que eram identificados. Em todos esses cenários além de justificar a sua escolha os alunos tinham que descrever quais medicamentos iriam prescrever para este paciente e a sua posologia. Além disso, eram estimulados a pensar no mecanismo de ação desses fármacos e explicarem seus possíveis efeitos colaterais, bem como manejar nesse contexto. Atividades como essa atuam como facilitadores para a aquisição do raciocínio clínico. Este por sua vez se refere ao processo cognitivo, através do qual o médico interpreta os sinais e sintomas apresentados pelo paciente com a finalidade de promover um diagnóstico para a partir dele estabelecer uma conduta terapêutica (PEIXOTO JM, et al., 2010). 
O professor que guia a simulação tem papel fundamental, pois age como um facilitador para o aluno, direcionando os participantes para os pontos mais importantes e pertinentes de cada assunto discutido. Além disso, o professor era o responsável por criar um ambiente de aprendizado saudável e para isso aplicava as técnicas de feedback mais apropriadas para cada momento, tendo como objetivo não constranger o aluno em nenhum momento para evitar a retração do mesmo nas próximas discussões (RESTREPO JD, et al., 2018).

Tendo em vista o momento de pandemia e a impossibilidade de realizar atividades práticas, o ambiente de simulação contribuiu para a fixação do conteúdo, além de promover maior segurança quanto raciocínio e conduta do medica em um ambulatório de saúde mental, nas patologias psíquicas mais prevalentes. Por meio da análise do comportamento do médico representado na simulação, os acadêmicos também puderam compreender as condutas éticas mais adequadas frente a um paciente psiquiátrico.

Dado o exposto, mesmo com a pandemia do COVID-19, que impôs várias restrições às atividades práticas presenciais, inviabilizando os estágios em saúde mental, houve um desenvolvimento significativo das habilidades de comunicação, senso crítico e raciocínio clínico. Sendo assim, a aplicação do debriefing no póssimulação remota se mostrou um artifício de aprendizagem eficaz.

Assim, os autores acreditam que essa metodologia possa ser um recurso para a manutenção do nível de qualidade do ensino, principalmente, em momentos da utilização do ensino híbrido. O ensino híbrido ganhou força com a pandemia e se mostra como uma grande tendência em um mundo cada vez mais digital, tendo em vista que durante esse período, esse modelo se mostrou viável para a criação de um ambiente de aprendizado integrado. Dessa forma, fica constatado que as ferramentas tecnológicas no contexto ensinoaprendizagem podem ser utilizadas não só para desenvolver o conteúdo teórico, como está sendo na maioria dos cursos de graduação por meio de aulas remotas, mas também podem ser manuseados para desenvolver habilidades humanas necessárias para uma efetiva prática médica. Por fim, fica evidente que quando utilizado de forma coerente, a tecnologia pode ser uma ferramenta eficaz para tapar uma lacuna que ocorre devido ao distanciamento do estudante do ambiente de prática médica.

\section{REFERÊNCIAS}

1- ABEER A, et al. Effective ness of Video-Assisted Debriefing in Health Education: An Integrative Review, 2018 ; 1 : 1420

2- BUI A, et al. Feedback Enhances Compliance with Operating Room Debriefs: Structured Feedback Enhances Compliance with Operating Room Debriefs. J Surg Res, 2021; 1: 425-432

3- CONOSCENTI E, et al. Post-crisis debriefing: A tool for improving quality in the medical emergency team system. Intensive and Critical Care Nursing, 2021; 4

4- D OSTA A, et al. Implementing Emotional Debriefing in Pediatric Clinical Education. Acad Pediatr, $2019 ; 4: 278$ - 282

5- FELIX H, et al. Debriefing Theories and Philosophies in Medical Simulation. Stat Pearls, 2020;10

6- FERRAZ AP, BELHOT R. Taxonomia de Bloom: revisão teórica e apresentação das adequações do instrumento para definição de objetivos instrucionais; vol.17, 2020;11

7- GRANT V, et al. Difficult debriefing situations: Atool box for simulation educators. Med Teach, 2018; 7: 703-712

8- NUNES S, HARDER N. Debriefing and Palliative Care Simulation. J Nurs Educ, v. 58,2019 ;10: 569-576.

9- PEIXOTO J, et al. Processos de Desenvolvimento do Raciocínio Clínico em Estudantes de Medicina, $2018 ; 11$

10-RAMNARAYAN G, et al. Examining the Effective ness of Debriefing at the Point of Care in Simulation-Based Operating Room Team Training, 2008;11

11-RESTREPO J, et al. Student 's Perception of Peer and Faculty Debriefing Facilitators Following Simulation- Based Education. J Allied Health, 2021; 7: 107-112

12-SANDRA J, et al. Simulation and Debriefing in Health Care Education: A Systematic Review. Journal of Nursing Education, 201; 6: 393-398.

13-SARAH B, et al. Facilitation and Debriefing in Simulation Education. Otolaryngologic Clinics of North America, 2017; 10: 989-1001.

14-SCHERTZER K, PATTI L. In Situ Debriefing in Medical Simulation. Stat Pearls, 2020; 9

15-TWIGG S. Clinical event debriefing: a review of approaches and objectives. Curr Opin Pediatric, 2020; 6: $337-342$ 